\title{
CASAS Y PALACIOS NAZARÍES. SIGLOS XIII-XIV. Antonio Orihuela Uzal.
}

\author{
Barcelona 1996. Colección El Legado Andalusí.
}

El objetivo del trabajo, que fue la tesis doctoral del autor, es realizar un análisis completo de todos los edificios de carácter residencial de época nazarí ( 1232- | 492) conocidos, sin distinción de orígen, tamaño, calidad artística ni estado de conservación, centrado principalmente en los aspectos tipológicos, funcionales, compositivos y constructivos.

Los criterios de selección del corpus de edificios estan razonados: todos aquellos restos que permitan reconstruir la planta. Se descartan ejemplares como el conjunto de los Guajares, por datarse en el momento de transición del poder almohade al nazarí y estar situado en ámbito rural, los situados al otro lado del Estrecho de Gibraltar tales como los de Belyounech y Qasr es-Seghir, pese a su semejanza con las viviendas granadinas, y otros donde las funciones militares se superponen a las propiamente residenciales como sucede en algunas torres de la Alcazaba de la Alhambra y la torre de Gabia, situada en La Vega granadina.

El análisis viene condicionado por los datos disponibles, de naturaleza muy heterogénea. Encontramos grupos con una problemática específica: a) edificios conservados y restaurados en distintas épocas b) edificios conservados sin restauracion donde se mantienen las modificaciones posteriores (sólo se incluye en este apartado la casa de los Gigantes en Ronda), c) restos excavados y consolidados, d) restos excavados y desaparecidos, e) restos excavados y soterrados.

De los 50 edificios catalogados la mitad procede de excavaciones arqueológicas, con ejemplares tan notables como los palacios de Abencerrajes y del Partal alto, Dar al-Arusa, parte substancial del Exconvento de S. Francisco y los conjuntos de las alcazabas de la Alhambra y de Málaga, casi todas llevadas a cabo durante la primera mitad de siglo. A la segunda mitad pertenecen los trabajos de Prieto Moreno en la Alhambra, la excavación del solar de la casa de los arquitectos en los años 60, o las que, según dicen algunos, dirigió Fernandez Puertas en el palacio de los Abencerrajes (hay que indicar que en su conjunto los trabajos de los años 90 en este mismo sitio se limitaron a la limpieza de lo ya excavado décadas antes). En general se han excluido datos provenientes de las excavaciones arqueológicas mas recientes, por ausencia de una publicacion.

El estudio también requiere el análisis previo de las restauraciones sufridas por las viviendas conservadas. No olvidemos que la misma Alhambra es resultado de una amplísima serie de intervenciones donde se yuxtaponen los criterios vigentes en cada momento. Como indica el autor su documentación exige tres niveles de lectura: el conocimiento de la obra original conservada en el momen- to de la intervención, del proyecto restaurador y, por último, de los trabajos realmente efectuados. De estos últimos no suele quedar constancia gráfica, cuando se trata de la restauración propiamente dicha.

El análisis crítico de la documentación gráfica procedente de restauraciones, y su comparación con los restos conservados da como resultado una amplia gama de matices y posibles enfoques metodológicos. Unificarlos para presentar un análisis de conjunto de un repertorio tan heterogéneo no es tarea sencilla. La planta es el único denominador común. Elaborar toda la planimetría, revisando las correspondiente a restos destruidos u ocultos y efectuar nuevos levantamientos -cuando ha sido posible- con técnicas fotogramétricas, es sin duda una de las aportaciones mas notables de este trabajo pues pone a disposición de los investigadores un material básico imprescindible. Se une a ello el conjunto de hipótesis restituidoras grafiadas, donde se contiene el análisis, valoración e interpretación de un amplio conjunto de problemas que superan incluso los límites del tema objeto de la tesis doctoral.

El estudio se divide en dos partes. La primera comprende los cinco primeros capítulos y abarca aspectos generales. El capitulo I corresponde al estudio tipológico. Tras una breve síntesis de los precedentes de los tipos básicos: patio de crucero, patio de dos pórticos y patio sin pórticos, se establece la clasificación tipológica de la arquitectura doméstica nazarí. Distingue dos tipos según la casa posea patio o carezca del mismo, dentro del primero los subtipos se fijan en función de la presencia lausencia de pórticos y de la organización en planta del patio: en crucero o con alberca central; en esta segunda no considera relevante ni el tamaño de la misma ni su desplazamiento hacia un lateral. En el subtipo sin pórticos, distingue entre grupo completo y elemental en base al número de elementos funcionales presentes. En las casas sin patio diferencia tres subtipos: qubba, torre palacio y el que denomina compacto. Deja bien claro que al tipo dominante: dos pórticos y alberca central, no tiene que el mas extendido en la ciudad y que su predominio entre la conservada se debe a su adcripción a una clase social alta.

El capítulo segundo está centrado en el estudio funcional, suministro y almacenamiento de agua, letrinas y saneamiento, baños privados, amplio apartado dedicado a las salas polifuncionales su origen y evolución, las tacas y alhacenas y, por último, los pórticos.

En el tercer capítulo se abordan los temas de composición arquitectónica: orientación, ejes compositivos, modos de combinación de elementos arquitectónicos, el sistema pórtico/sala alargada/qubba y sus variantes, albercas y arriates con clasificación de las variantes encontradas, y los alzados. Es capitulo que admite ser ampliado 
con generosidad incluyendo otros elementos. Alguno, como las celosías, fue redactado por el autor con posterioridad a la lectura de la tesis doctoral aunque no ha sido incluido en la obra impresa.

El capítulo IV estudia las técnicas constructivas. Cimientos, muros, alfarjes y armaduras, tejados y aleros, escaleras y soportes. No encontramos referencia pormenorizada a los acabados de superficies: decoraciones pintadas, yeserías y alicatados, ni a las distintas modalidades de solería.

Buena parte del contenido de dichos capítulos ya fue avanzado en lo esencial en su trabajo sobre los inicios de la arquitectura residencial nazarí, publicado en otro volúmen de la misma colección (Casas y palacios de al-Andalus. Siglos XII-XIII).

En capítulo $V$ se resume la historia de la conservación de la arquitectura residencial nazarí. Distingue varias fases: la restauración romántica decimonónica, una etapa de transición representada por Modesto Cendoya, que da paso a Leopoldo Torres Balbás, sigue la etapa que define como de aislamiento cultural con Francisco Prieto Moreno como figura dominante, y por último la que se abre con la tranferencia de competencias a la Junta de Andalucía en 1984. Es tema importante que reclama monografía específica. Este capítulo debe ser completado con los epígrafes dedicados a la restauración en cada uno de los edificios o conjuntos descritos en la segunda parte del libro. Los numerosos datos recopilados enriquecen de manera notable una historia ignorada hasta fechas muy recientes y que presenta episodios oscuros que deben ser objeto de una rigurosa documentación; valga de ejemplo los numerosos datos sobre la etapa Prieto-Moreno, que, a nuestro entender, configuran uno de los períodos mas negros en la conservación de la arquitectura nazarí, durante el cual se arrasaron los restos del palacio ocupado por el Convento de S. Francisco no incluidos en el mismo para levantar el parador de turismo, lo mismo sucedió con la casa en la calle Real para darle acceso al nuevo Parador, o con la casa junto a la mezquita mayor de la Alhambra para ubicar los transformadores. En este período se produjo el hallazgo del Palacio de los Abencerrajes, cuyas circunstancias aun no estan aclaradas y donde las evidencias de haber sido arrasado en época reciente son numerosas, y la reconstrucción para su uso como museo de las viviendas encontradas en la Alcazaba de Málaga.

Cabría en esta primera parte mayor exhaustividad en la enumeración de los componentes morfológicos y soluciones estructurales tales como los encuentros de alfarjías con los muros, solución de aleros, encuentros de arquerías con los muros, solución de huecos en muros con distintas técnica constructiva, tecnicas y materiales utilizados en los muros en base a su grosor o función estructural, preparación de pavimentos, etc.. Algunos de dichos elementos admiten analisis en una doble dimensión morfológica y constructiva, así los arcos. El arco abarcante en Alcázar Genil para resolver los acesos a las salas laterales o la solución de dicho acceso en el Cuarto Real de St. Domingo son ejemplos de modos que contrastan con lo visible en los palacios alhambreños y que justificarían su sistematización. Aunque son datos que se encuentran recogidos en la segunda parte de la obra, en la planimetría, texto o documentación fotográfica, pudieran haberse agrupado en los capítulos iniciales.

La propuesta de clasificación tipológica admite, a nuestro parecer, algunos matices. Si tenemos en cuenta la cronología cabría distinguir un subgrupo formado por Abencerrajes, Partal Alto, Generalife, Exconvento de S. Francisco, caracterizado por el predominio de esquemas planimétricos derivados del patio de crucero evolucionado. Si además consideramos el patio de crucero del Palacio de los Leones y descartamos Dar al-Arusa, cuya alberca correspondería a un segundo momento funcional, el patiotipo representado por el Palacio de Comares, al que nadie discute su relevancia como principal creación nazarí, parece mas excepción que norma en el conjunto de palacios nazaríes conservados. Es un tema sobre el que conviene profundizar.

El tipo qubba es sin duda el mas conflictivo. Agruparía el Partal, Cuarto Real de St. Domingo y Alcázar Genil. Aunque se justifica por el estado actual de los edificios no debemos olvidar que se trata de elementos cuyo contexto espacial ha sido fuertemente alterado. Lo insólito de la organización puesta al descubierto en las excavaciones del Cuarto Real de St. Domingo muestra que salvo que un reconocimiento arqueológico de dicho contexto elimine incertidumbres, su posible naturaleza de fragmentos pertenecientes a conjuntos desaparecidos debe ser tenida en cuenta.

La segunda parte está constituida por el catálogo, que constituye el núcleo central del trabajo. Los treinta y cinco grupos estudiados se dividen en cinco apartados: a) Alhambra, con 19 conjuntos; b) alrededores de la Alhambra, que abarca el Generalife, la Casa de los Amigos -que aunque pertenece al mismo complejo se considera como entidad autónoma-, y Dar al-Arusa; c) Casco urbano de Granada, con nueve ejemplares; d) Alrededores de la ciudad, con Alcázar Genil y Darabenaz; e) Provincia de Málaga, con el conjunto de la Alcazaba malagueña y la casa del Gigante en Ronda. El riguroso esquema expositivo facilita la lectura y la posibilidad de establecer comparaciones entre grupos y elementos. Un primer apartado sobre emplazamiento contextualiza el edificio, a continuación se analiza la historiográfia y sitúa el estado de la cuestión de los datos existentes y las distintas valoraciones dadas por los investigadores; se indica también la planimetría existente y el archivo donde se encuentra. Le sigue una descripción ajustada a las necesidades y problemática de cada conjunto, donde se unifican los datos positivos reconocibles con las propuestas interpretativas. Finaliza con la reseña pormenorizada de las distintas reformas y restauraciones conocidas. Cuando las características del edificio lo requieren se incluyen otros apartados. La caracterización de los elementos es precisa. Son numerosas las novedades aportadas tanto en datos positivos como en la indicación de problemas puntuales. Señalamos algunas: en el 
palacio de los Abencerrajes se plantea una posible organización de la torre central que explicaría la ubicación de los paños de yesería conservados en el Museo de la Alhambra; en el Partal se presenta una relación entre el pórtico y el mirador de la parata Sur que define unas relaciones espaciales indispensables para entender el ámbito específico del palacio; en el Palacio de Comares el análisis del cuerpo Sur del Patio de los Arrayanes permite descartar la existencia de una sala intermedia y rastrea los elementos que pueden ayudar a restituir el sistema de relaciones espaciales originales, tanto en horizontal como en vertical; en el Partal Alto (palacio de Yusuf III) la hipótesis de restitución planimétrica ayuda a comprender la importancia de espacios que permanecen sin investigar dentro del recinto alhambreño y que en el futuro puede dar lugar a sorpresas espectaculares; en las casillas del Partal se expone cómo son resultado de una progresiva densificación espacial; en la casa bajo el jardín de la calle Real se restituye una vivienda con baño, cuya relación con el parcelario existente hasta los años 60 y con el inmediato palacio de los Abencerrajes ayuda a entender las distintas transformaciones que ha sufrido la zona, tanto en época medieval como moderna. Destaquemos tambien el esfuerzo en hacer legibles restos tan problemáticos como el palacio del Secano, la casa bajo la antigua del arquitecto de la Alhambra, inmediata a la Puerta del Vino o el palacio de Dar al-Arusa.

Se apuntan las dudas adecuadamente y el carácter hipotético de las soluciones ofrecidas en base a su adcripción tipológica cuando es necesario. La complejidad de algunos temas y una posible valoración diferente de ciertos datos puede abrir en el futuro una discusión, por otra parte necesaria.

Tema interesante por su novedad, ampliamente expuesto a traves de los distintos epígrafes del catálogo es el problema de las planta altas, su presencia, ubicación, extensión y sistema de accesos. Plantea A. Orihuela la existencia de un grupo donde los cuerpos menores, porticados, con salas desarrolladas en doble altura carecerían de planta alta mientras que los lados mayores mayores, laterales, dispondrían de algorfa. Todos los frentes quedarían enlazados por un alero a la misma altura. En un segundo momento, ante las presiones demográficas existentes en Granada se produciría la construcción de plantas altas sobre los frentes porticados. Se establecen tres categorías de edificios: a) viviendas construidas desde un principio con planta alta sobre el pórtico, b) viviendas a las que en un momento tardío nazarí se les añade una planta, c) viviendas a las que se añaden galerías en época morisca. La distinción implica metodologías propiamente arqueológicas, algo que esperamos que sea adecuadamente tenido en cuenta en futuros proyectos de restauración de las tipologías abarcadas por el problema: las casas nazaríes y las moriscas.

El grupo de ejemplares con galerías sobre los pórticos está constituido por el palacio de Daralhorra, la almunia de Darabenaz, la Casa de los Infantes y el cuerpo Sur del Patio de los Arrayanes. Todos con arcos de yeso sobre pilares de ladrillo salvo la última que posee colum- nas. La necesidad de hacer corresponder los ejes de los soportes de ambas plantas, la menor altura de la superior, y la diferencia de luz entre el vano central y los laterales crea un problema que explica la solución del Patio de Arrayanes donde se recurre a dejar adintelado el hueco central. En el Convento de Zafra se plantean dudas sobre su existencia en un primer momento. En el Cobertizo de St. Inés (cuerpo Norte) se piensa en añadido posterior, quizá del siglo XV.

Dos soluciones específicas de los cuerpos altos correspondientes a las crujías menores se encuentran en el cuerpo Sur del patio de los Arrayanes y en la casa del Cobertizo de St. Inés (cuerpo Sur). En la primera la galería intermedia ofrece una manera de resolver el nivel de la galería superior cuando se presenta desproporción entre la altura del pórtico y la sala baja. La algorfa del Cobertizo de St. Inés muestra una disposición con pequeñas ventanitas enrasadas con el pavimento que cabe relacionar con el cuerpo alto de la fachada del Palacio de Comares. Otra forma interesante de resolver el tema se encuentra en el cuerpo Sur del Generalife, donde lo insólito respecto a lo característico nazarí se impone, tanto en el mismo pórtico como en la entreplanta y accesos a la sala alta, aunque su estado de conservación hace imposible reconocer la solucion original.

Fernandez Puertas en su momento apuntó no solo la existencia de plantas altas sobre los cuerpos porticados sino también dobles escaleras para acceder a cada uno de ellos, algo que no justifica la planimetría de las viviendas. La presencia de escaleras no solucionaría el problema pues en la propuesta de A. Orihuela sólo darín acceso a los cuerpos laterales, donde sí existirían plantas altas.

Se rechaza la existencia en época nazarí de galerías sobre pies derechos de madera y también de otras laterales para comunicar los frentes porticados, opción que sería propia del momento morisco, optando por el uso de una de las crujías laterales como pasaje de comunicación.

En cuanto a posibles influencias externas, ausentes las referencias a la arquitectura magrebí, salvo para temas muy puntuales, encontramos dos a la arquitectura egipcia: a Fustat a propósito del origen y desarrollo de lo que Creswell definiera como planta en T, y a la posible derivación de la qaca fatimi a propósito de la Torre de las Infantas, según la teoría expuesta por Torres Balbás en su estudio sobre las salas con linterna central, donde en nota señalaba una influencia de la arquitectura egipcia en la nazarí mayor de la que había reconocido en estudios anteriores. Es tema interante y complejo que admite amplio desarrollo desde las investigaciones mas recientes como las excavaciones de G. Scanlon en Fustat y la síntesis de A. Ostrasz sobre su arquitectura, de análisis como el que plantea Hazem I. Sayed sobre su evolución en la arquitectura egipcia, y del estudio de su influencia en Occidente, con datos como los que apunta L. Golvin a propósito de la Qubba de Palermo. Pero además de esta línea, que afecta sobre todo a su consideracion como arquitectura palatina, pensamos que las torres de la Cautiva y de las Infantas muestran en sus soluciones adinteladas y ambitos porticados en torno a espacios centrales un 
esquema facilmente transponible a patios, donde puede presentarse con total ausencia de elementos decorativos, lo que implica la posible existencia de una tipología no identificable a través de los edificios conservados. El interés de este tema radica en que podría explicar la transición a los esquemas moriscos, incomprensibles en el contexto de una tradicion local si nos atenenemos a las conclusiones del trabajo que comentamos. Ni que decir tiene que es un problema que corresponde ser resuelto por la arqueología urbana.

La determinación cronológica del grupo como nazarí viene dada en buena parte por su ubicación en el complejo alhambreño. Del medio centenar de viviendas estudiadas, 37 pertenecen al complejo palatino de la Alhambra y su entorno. Constituyen base sólida para definir un marco cronológico de referencia a la hora de caracterizar como nazaríes las correspondientes tipologías, sin confusion con permanencias de períodos históricos anteriores. Hay pues un grado de precisión razonablemente alto a la hora de establecer características atribuibles a un determinado momento histórico. El momento inicial comprende un conjunto de veinte edificios levantados durante reinado de los sultanes Muhammad II y Muhammad III, es decir hasta 1309, que ya fue presentado por el autor en un trabajo incluido en otro volúmen de la misma colección. No se incide en la justificación o no de los criterios por los que Fernandez Puertas distinguió entre el grupo atribuible a Muhammad II (Cuarto Real de St. Domingo, Palacio de Abencerrajes y Partal Alto) y Muhammad III (Convento de S. Francisco, Generalife, Partal y Casa junto al baño de Polinario). El momento final queda diluido. Digamos a este respecto que el gran problema planteado en la arquitectura nazarí es su relación con el grupo de casas moriscas, que no sería mas que epígono de la tradición nazarí.

En cuanto a los procesos de modificación espacial y estructural reconocibles, aunque sólo en tres ejemplares (Darabenaz, Casa del Marqués del Cenete y Casa de los Gigantes en Ronda) la planimetría recoge precisiones sobre la cronología de los muros, en el texto se indican numerosos ejemplos de dichas transformaciones. El Generalife es muy significativo a este respecto. La decoraciones superpuestas en el mirador central en el patio de la acequia, los arcos de acceso a la sala norte y la qubba añadida a dicha sala marcan fases dentro de un único conjunto arquitectónico. También se apunta la superposición de una zona del Generalife sobre la casa de los Amigos. En la Casa de los Girones tambien existen dos decoraciones pintadas superpuestas. En Cobertizo de St. Inés se indica el posible añadido de una planta alta, dentro del mismo período nazarí, vendría dado por un cambio en el tapial correspondiente a dicha zona; en la casa del Convento de Zafra serían dos las reformas introducidas, por un lado la posible existencia de un pórtico de cinco vanos en la crujía Sur, luego reducido a tres por incorporación de una crujía lateral, por otro, el descentrado de los pilares de la galería alta sería indicio de su reconstrucción, unido a la reforma de los arcos del pórtico correspondiente. Plantea la propuesta apuntada por R. Manzano de una construcción tardía del cuerpo Sur del patio de los Arrayanes, en el reinado de Muhammad $\mathrm{V}$, sobre estructuras anteriores. En Dar al-Arusa se indican las dos fases constructivas identificadas. Ya aludimos a al conjunto de las casillas del Partal. En la casa inmediata al Palacio de los Abencerrajes se indica las dos fases constructivas. Aparte, la problemática de las posibles modificaciones introducidas en época morisca.

Hay un último aspecto sobre el que un trabajo como este: una revisión global de la arquitectura doméstica nazarí, nos invita a reflexionar. La sistematización tipológica se refiere a complejos donde no es posible efectuar registros estratigráficos e incluso donde la sustitución de los materiales constructivos originales puede hacer que no exista ningún elemento material correspondiente a la cronología del momento histórico fundacional, aunque los espacios conservados sí pudieran corresponder a la distribución primitiva. Se trata además de estructuras y espacios que se han visto sometidos a los criterios restauradores a veces arbitrarios. La metodología arquitectónica aplicada a dichos contextos presenta el peligro de un reduccionismo tipológico que anule las especificidades históricas no racionales, hecho que llevado a su extremo conduce a la aberración de corregir la historia. Los criterios de racionalización utilizados en algun momento en la restauración de los edificios nazaríes encuentran nítidos ejemplos en el trabajo. La sustitución de los pilares de ladrillo por columnas en el Partal, pese a los problemas interpretativos existentes, es un ejemplo de imposición de lo que debe ser sobre el edificio conservado. En el palacio de Daralhorra, se pensó en trasladar la alberca de sitio porque no respondía a la norma que debería seguir la casa nazarí y no se dudaba en destruir lo existente para restituir una hipotética imagen históricamente correcta. Comparese el plano 76, una sección de la casa de la calle Horno de Oro, con la figura 480 de la Historia de la Arquitectura Española de Chueca Goitia de 1965, donde se reproduce la misma sección, para ver cómo se ha desmontado el muro de fachada y rebajado el alero con canecillos nazaríes para alinearlo con la rasante de la galería interior; este edificio ha sido virtualmente desmontado y vuelto a reedificar en sucesivos proyectos de restauración, por lo que las evidencias materiales de su posible orígen nazarí y remodelación morisca han desaparecido, y por si era poco, la última intervención no se ha resistido a incluir un toque de diseño forrando de marmol blanco su alberca. En la Alcazaba de Málaga los problemas interpretativos derivados de una reconstrucción excesiva son irresolubles.

Actuaciones semejantes sólo son posibles desde la idea de un conocimiento acabado y total de todas las variables existentes en la arquitectura de un momento histórico. Sin embargo parece necesario resaltar que la arquitectura doméstica nazarí conocida no puede ser identificada con la totalidad de la casuística correspondiente a dicho momento histórico. La absoluta ausencia de viviendas correspondientes al momento inmediato anterior -siglo XII-, en la ciudad de Granada debería ponernos en guardia, pues viene a decirnos que desconocemos el grado de permanencia de tipología anteriores durante el sultanato nasrí, y su vigencia junto a nuevas tipologías, como 
tampoco conocemos la influencias de otras comunidades musulmanas, tanto norteafricanas como -ipor qué no?asentadas en territorio cristiano, es decir, de los mudéjares propiamente dichos en el desarrollo de nuevos usos residenciales. Ejemplos de esta problemática pueden ser rastreados en el trabajo de $\mathrm{A}$. Orihuela. En la Casa del Convento de Zafra algunos elementos (una serie de mechinales que debieron corresponder a maderos de pequeña sección) parecen indicar una determinada organización inicial, aunque la misma se considera "poco sostenible por lo anómalo de tal disposición", teniéndose que reconocer que se carecen de datos o de posibles paralelos que aclaren la razón de la existencia de tales elementos. La disposición encontrada en las excavaciones arqueológicas del Cuarto Real de St. Domingo hubiera sido imposible plantearla como hipótesis de trabajo, puesto que carece de paralelo en la arquitectura nazarí. Como también es insólita la organización de la crujía Sur de la casa en el Cobertizo de St. Inés. Son ejemplos de "anomalías" que ponen en evidencia los límites de nuestros conocimientos.

Con todo esto queremos decir que las propuestas restituidoras apuntadas en este trabajo, en cuanto defí- nen sólo hipótesis basadas en el repertorio conocido, deben entenderse como instrumentos de trabajo para una verificación posterior, ya sea en el propio edificio, cuando es posible, o por otros hallazgos posteriores que permitan valorar el grado de generalización de una determinada solución espacial o constructiva. En este sentido dichas propuestas restituidoras articulan los términos de un posible diálogo con la investigación arqueológica que abre unas espectativas de investigación que deben ser adecuadamente desarrolladas en el futuro.

En resumen, se trata de un trabajo de una gran densidad que admite lecturas a distintos niveles. Los múltiples datos que evidencian una lectura atenta y directa de los edificios analizados, junto a una completa planimetría y la incidencia en una interesante problemática hasta ahora ausente en las investigaciones sobre arquitectura nazarí, hacen de este trabajo un instrumento imprescindible para quienes se interesan en el estudio de la vivienda hispanomusulmana, y la arquitectura nazarí en especial.

José Antonio García Granados 